\title{
Spin-Orbit Electric Field Gradient in Cubic Ferromagnets: Strong Magnetization-Direction Dependence of the Field Gradient Distribution
}

\author{
G. Seewald, E. Hagn, and E. Zech \\ Physik-Department, Technische Universität München, D-85748 Garching, Germany \\ R. Kleyna and M. Voß \\ Institut für Physikalische und Theoretische Chemie, Universität Erlangen-Nürnberg, \\ D-91058 Erlangen, Germany \\ D. Forkel-Wirth and A. Burchard \\ PPE Division, CERN, CH-1211 Geneva 23, Switzerland \\ The ISOLDE Collaboration \\ CERN, CH-1211 Geneva 23, Switzerland \\ (Received 19 March 1998; revised manuscript received 19 October 1998)
}

\begin{abstract}
The electric quadrupole interaction of ${ }^{191} \mathrm{Pt}\left(I^{\pi}=3 / 2^{-} ; T_{1 / 2}=2.9 \mathrm{~d}\right)$ in an Fe single crystal was measured for magnetization $M$ parallel to the crystallographic [100], [110], and [111] axes. For $M \|$ [111] and [110] the distribution of the electric field gradient (EFG) is relatively sharp. For $M \|[100]$ the EFG distribution is by a factor of 2.8(6) broader than for $M \|[111]$. Additional data on $\mathrm{Os}, \mathrm{Re}, \mathrm{Ir}$, and $\mathrm{Au}$ in $\mathrm{Fe}$ indicate that this behavior seems to be of general nature: In all cases the EFG distribution for $M \|$ [100] is considerably broader than for $M \|$ [110] and [111]. [S0031-9007(98)08187-3]

PACS numbers: 76.60.Jx, 75.50.Bb, 76.60.Gv, 76.80.+y
\end{abstract}

At the nuclear site of heavy impurities in the cubic ferromagnetic host lattices $\mathrm{Fe}$ and $\mathrm{Ni}$ an electric field gradient (EFG) exists in addition to the magnetic hyperfine field. There have been many investigations for the understanding of this EFG, both experimental [1-12] and theoretical [5,13-16]. It was first observed by Aiga and Itoh with nuclear magnetic resonance (NMR) measurements on ${ }^{191} \mathrm{Ir}$ and ${ }^{193} \mathrm{Ir}$ in polycrystalline $\mathrm{Fe}$ and Ni [1,5]. Shortly afterwards it was confirmed by Mössbauer-effect measurements on ${ }^{193} \mathrm{Ir}$ in $\mathrm{Fe}$ and $\mathrm{Ni}[2,3]$. For the system $\mathrm{AuFe}$ the EFG could also be detected with spin echo measurements on ${ }^{197} \mathrm{AuFe}[8,9]$.

In principle, the EFG in a cubic ferromagnet can arise from magnetostriction and from unquenched local moments. As the observed EFG's are much larger than an EFG due to magnetostriction could be, it was early adopted that the main source is the unquenched orbital momentum [13-15]. From theoretical calculations Demangeat [13] postulated that EFG's originating from an unquenched orbital momentum should strongly depend on the direction of magnetization with respect to the crystallographic axes. Based on experimental results of NMR measurements on ${ }^{191,193}$ Ir in Fe single crystals [5] and NMR on orientated nuclei (NMR-ON) measurements on ${ }^{192} \mathrm{Ir}$ in a Ni single crystal [4] it was concluded, however, that such a dependence does not exist. Another theoretical approach was considered by Gehring and Williams [15]. In the general framework of their model, the EFG may, depending on the relative sizes of spin-orbit coupling, the crystal field, and the exchange splitting, depend on the direction of the mag- netization with respect to the crystal axes, or not. Led by the experiments suggesting the EFG to be invariant, they concluded that the effects of the crystal field can be neglected, which leads to an invariant EFG.

Recent NMR-ON and modulated adiabatic fast passage on oriented nuclei (MAPON) measurements on $Z=77$

${ }^{188} \mathrm{Ir}$ in an $\mathrm{Fe}$ single crystal showed a strong dependence of the EFG on the direction of magnetization with respect to the crystallographic axes [11]. In these experiments no special attention was directed to the widths of the EFG distributions, as there was no theoretical hint that the EFG width could be directional dependent. The measured ratio, $\Gamma^{([100])} / \Gamma^{([111])}=1.5(5)$, showed, however, that the width could be larger for $M \|$ [100] than for $M \|$ [111]. On the other hand, these data were not conclusive because of the too large statistical error. Thus, it was concluded that the EFG is sharp and that the distribution is introduced by the nonperfect surface quality of the used single crystal. Subsequent MAPON measurements on $Z=79{ }^{198} \mathrm{Au}$ in Fe showed a similar crystallographic-direction dependence of the EFG [12]. Concerning the EFG widths, the ratio $\Gamma^{([100])} / \Gamma^{([111])}=1.4(4)$ again did not allow one to draw strong conclusions.

In this Letter we show that, in contrast to ${ }_{77} \mathrm{Ir}$ and ${ }_{79} \mathrm{Au}$, the EFG of $Z=78 \mathrm{Pt}$ in Fe has completely different features: For magnetization in the [111] and [110] directions the EFG is relatively sharp with a unique negative sign, whereas for [100] the EFG is broadly distributed around an average value near zero. Such a behavior had not been expected. 
In the case of a hyperfine interaction consisting of a magnetic dipole and an electric quadrupole interaction (EQI) the resonance spectrum for NMR consists of a set of $2 I$ equidistant subresonances, where $I$ is the nuclear spin. The quadrupole subresonance separation $\Delta \nu_{\mathrm{Q}}$ is given by

$$
\begin{aligned}
\Delta \nu_{\mathrm{Q}} & =3 \nu_{\mathrm{Q}} /[2 I(2 I-1)], \\
\nu_{\mathrm{Q}} & =e Q V_{z z} / h,
\end{aligned}
$$

where $\nu_{\mathrm{Q}}$ is the quadrupole interaction frequency, $e Q$ is the spectroscopic quadrupole moment, and $V_{z z}$ is the principal component of the traceless EFG tensor.

In this work the techniques of adiabatic fast passage on oriented nuclei (AFP-ON) and MAPON were applied for precise measurements of the quadrupole interaction. In both methods the rf frequency is slowly and continuously swept through the resonance conditions. The interaction with the nuclear spin system is detected via the anisotropy of $\gamma$ radiation emitted in the decay of low-temperature oriented nuclei.

With AFP-ON on a quadrupole split system, cyclic permutation of the sublevel populations $a_{m}$ is obtained. For a quadrupole-split system with a unique sign of the quadrupole splitting, the final state after an AFP pulse is strongly different for the two different rf sweep directions: The sublevel population of the first accessed sublevel is carried through to the last sublevel, and the remaining $2 I$ populations are displaced one. This means that the sign of the quadrupole interaction can be determined with AFPON. In addition, via the detailed analysis of the time dependence of the $\gamma$ anisotropy after AFP pulses for both sweep directions, the fractional contributions of positive and negative quadrupole splittings can be determined uniquely.

The principle of MAPON is described in detail in Refs. [17,18]. A MAPON pulse consists of a sequence of two AFP pulses which are separated in frequency by $\Delta \nu$. As the influence of the MAPON pulse on the time evolution of the sublevel populations is different for $\Delta \nu>$ $\Delta \nu_{\mathrm{Q}}$ and $\Delta \nu<\Delta \nu_{\mathrm{Q}}$, the distribution of the EQI can be determined by differentiation of a MAPON spectrum $(\gamma$ anisotropy as a function of $\Delta \nu$ ).

The single-crystal ${ }^{191} \mathrm{Pt} F e$ sample was prepared as follows: An Fe single-crystal slice was cut from a singlecrystal rod, the surface normal being parallel to a [110] axis. The [100], [110], and [111] crystallographic axes then lie in the plane of the surface of the sample. Thus, by rotating the crystal in its position at the cold finger of the dilution refrigerator, the magnetization can be forced parallel to the [100], [110], and [111] axes with an external magnetic field. Much attention was paid to an extremely high surface quality. Cleaning and characterization of the surface were performed in a commercial VG ESCALAB 200 UHV chamber. After mechanical polishing, the surface was cleaned by repeated cycles of $\mathrm{Ar}^{+}$ion sputtering at 300 and annealing at $830 \mathrm{~K}$. The concentration of impurities and the ordering of the surface was controlled by x-ray photoelectron spectroscopy and low energy electron diffraction. Radioactive ${ }^{191} \mathrm{Hg}$ was produced via the $(p, 3$ pxn $)$ reaction on a liquid $\mathrm{Pb}$ lead target with the $1 \mathrm{GeV}$ CERN PS-booster proton beam, and implanted into the single crystal with $E=60 \mathrm{keV}$ at the mass separator ISOLDE. The implanted nuclei (total number $\sim 1 \times 10^{12}$; size of the beam spot $\sim 1.5 \times 3 \mathrm{~mm}^{2}$ ) are distributed within a layer of $\sim 5 \mathrm{~nm}$ width in a depth of $\sim 25 \mathrm{~nm}$ underneath the crystal surface. The impurity concentration in this layer is $\sim 0.05$ at. \%; i.e., the criteria for a dilute alloy are well fulfilled. ${ }^{191} \mathrm{Pt}$ is obtained as the daughter isotope in the decay cascade ${ }^{191} \mathrm{Hg}\left(T_{1 / 2}=0.8 \mathrm{~h}\right) \rightarrow$ ${ }^{191} \mathrm{Au}\left(T_{1 / 2}=3.2 \mathrm{~h}\right) \rightarrow{ }^{191} \mathrm{Pt}\left(T_{1 / 2}=2.9 \mathrm{~d}\right)$. All measurements were performed at millikelvin temperatures using a ${ }^{3} \mathrm{He}-{ }^{4} \mathrm{He}$-dilution refrigerator with a top-loading facility. The final measurements presented here were performed after annealing at $1070 \mathrm{~K}$.

MAPON measurements were performed for $B_{\text {ext }} \|$ [100] $\left(B_{\text {ext }}=2 \mathrm{kG}\right),[110]\left(B_{\mathrm{ext}}=3 \mathrm{kG}\right)$, and [111] $\left(B_{\text {ext }}=4 \mathrm{kG}\right)$. In all cases the magnetization was fully aligned by the external magnetic field. The distributions of the EQI as obtained from the MAPON spectra by least-squares fit are illustrated in Fig. 1. The "integral" sign of the EQI was measured with AFP-ON (see below); it is positive in all three cases. The EQI distributions as obtained from the least-squares fit imply that the

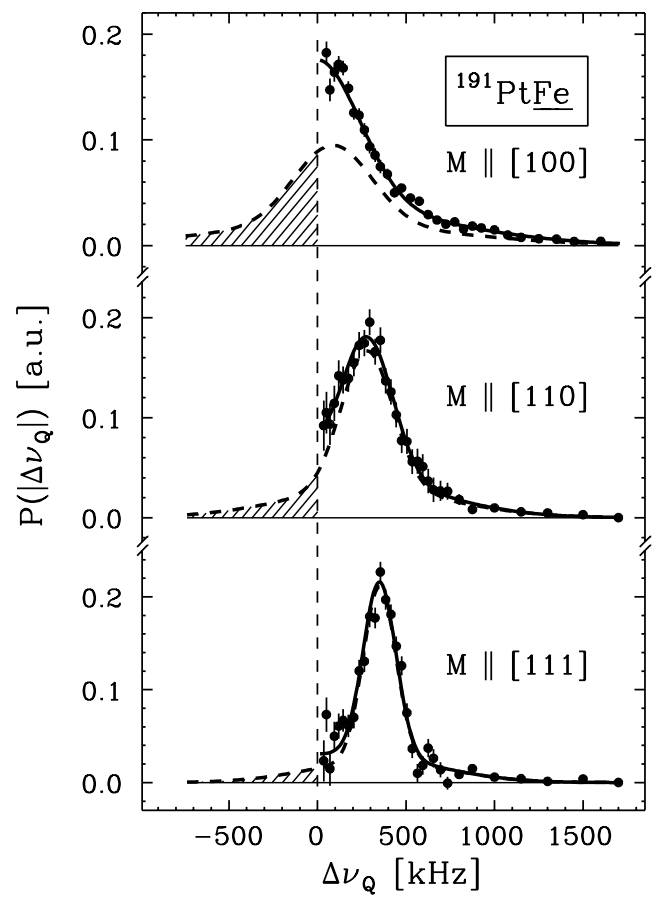

FIG. 1. Derivatives of the MAPON spectra for ${ }^{191} \mathrm{Pt} F e$ which represent the sign-independent distributions of the quadrupole splittings. The dashed lines represent the distributions of the quadrupole splitting as obtained from the least-squares fit. The sign-independent distributions are obtained by adding the positive and negative contributions (full lines). The shaded areas indicate the contributions with negative sign. 
fractional contributions of negative quadrupole splittings (shaded areas in Fig. 1) are 0.39(11), 0.13(5), and 0.08(5) for magnetization parallel to the [100], [110], and [111] directions, respectively. Whereas the measurements for $B_{\text {ext }} \|$ [110] and [111] show almost the "normal" behavior - a relatively sharp quadrupole interaction with unique sign - the measurement for $B_{\text {ext }} \|[100]$ demonstrates that in this case the EQI is given by a broad distribution, with positive and negative contributions.

The unexpected result on the large fractional contribution of negative quadrupole splittings for the [100] direction has been verified with AFP-ON measurements, which are shown in Fig. 2. The solid lines are the results of leastsquares fits in which the Korringa constant $C_{\mathrm{K}}$ for the nuclear spin-lattice relaxation, the adiabatic parameter $A$ for the AFP inversion, and the fractional contributions of negative quadrupole splittings were free parameters. The latter were found to be 0.38(3), 0.09(3), and 0.04(3), for [100], [110], and [111], respectively. The excellent agreement with the MAPON results can be viewed as justification for the EQI distribution used for the interpretation of the MAPON spectra.

The EQI frequencies in the maximum of the distribution are obtained by $\nu_{\mathrm{Q}}=2 \Delta \nu_{\mathrm{Q}}$ for $I=3 / 2$. The final results

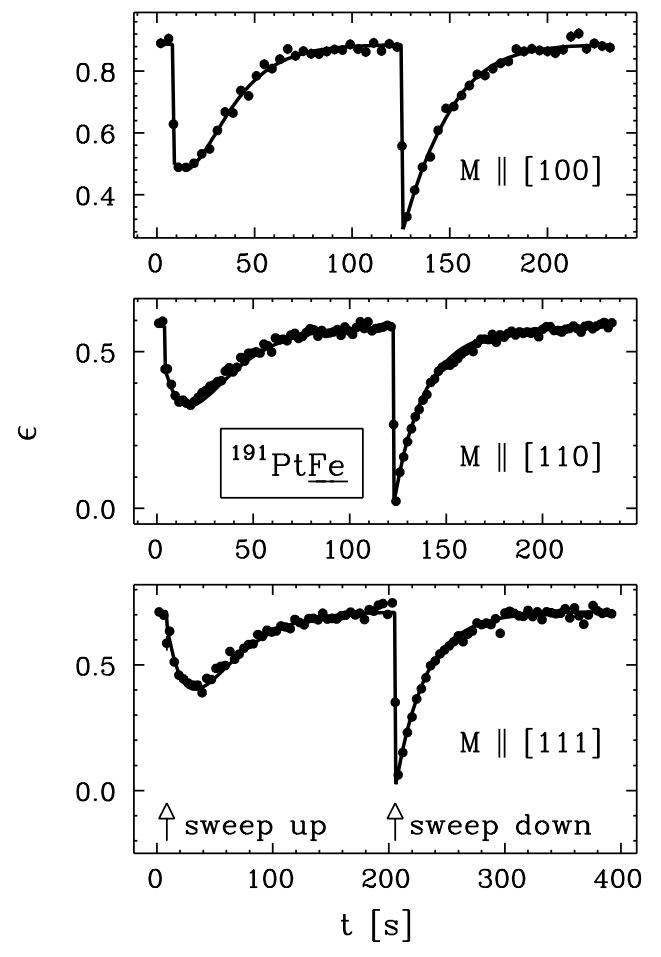

FIG. 2. AFP-ON spectra for ${ }^{191} \mathrm{Pt} F e$. The strongest asymmetry between sweep-up and sweep-down occurs for $M \|$ [111] due to the unique sign of all contributions to the quadrupole interaction, which is positive. This asymmetry is reduced for $M \|$ [100] indicating a considerable amount of negative contributions to the quadrupole interaction. The theoretical curves as obtained from the least-squares fits describe the experimental data perfectly. are $\nu_{\mathrm{Q}}^{([100])}=+0.25(9) \mathrm{MHz}, \nu_{\mathrm{Q}}^{([110])}=+0.60(2) \mathrm{MHz}$, and $\nu_{\mathrm{Q}}^{([111])}=+0.72(2) \mathrm{MHz}$. (The result for $\nu_{\mathrm{Q}}^{([100])}$ takes into account the ratio of positive and negative contributions obtained with AFP-ON.) Taking $Q\left({ }^{191} \mathrm{Pt}\right)=$ $-0.87(4) \mathrm{b}$ [19], the absolute values of the EFG in the maximum of the distribution are $-0.012(4),-0.029(2)$, and $-0.035(2) \times 10^{17} \mathrm{~V} / \mathrm{cm}^{2}$ for the [100], [110], and [111] directions, respectively. These are illustrated in Fig. 3, together with the EFG's for Re, Os, Ir, and Au in Fe $[11,12]$.

The width of the EFG distribution is 0.058(12), 0.037(4), and $0.021(2) \times 10^{17} \mathrm{~V} / \mathrm{cm}^{2}$ for the [100], [110], and [111] directions, respectively; i.e., the [100] width is by a factor of 2.8(6) larger than the [111] width. This cannot be due to experimental reasons as the measurements were performed on the same sample by just rotating the single crystal in its position on the cold finger of the cryostat.

Figure 4 shows the ratio of EFG widths $\Gamma^{([100])} / \Gamma^{([111])}$ vs the EFG anisotropy for Re, Os, $\mathrm{Ir}, \mathrm{Pt}$, and $\mathrm{Au}$ in $\mathrm{Fe}$. The following is evident: (i) The width for $M \|$ [100] is larger than the width for $M \|$ [111] in all cases. Assuming that the increase of the width is independent of the EFG anisotropy, we get as average value $\left\langle\Gamma^{([100])} / \Gamma^{([111])}\right\rangle=$ 1.84(17) (dotted line; the shaded area represents 1 standard deviation), which is different from 1.0 with statistical significance. (ii) The increase of the [100] width might be correlated with the EFG anisotropy (full curve in Fig. 4).

Concerning the EFG anisotropy (see Fig. 3) the following is evident: The sign of the anisotropy $\left(V_{z z}^{[100]}-\right.$ $\left.V_{z z}^{[111]}\right) / V_{z z}^{[111]}$ is positive for Re, Os, Ir, and Au, whereas it is negative for Pt. At present we can give no explanation for the sign change for Pt. Within the theoretical calculations of Refs. $[5,13,15]$ there is no indication for such a

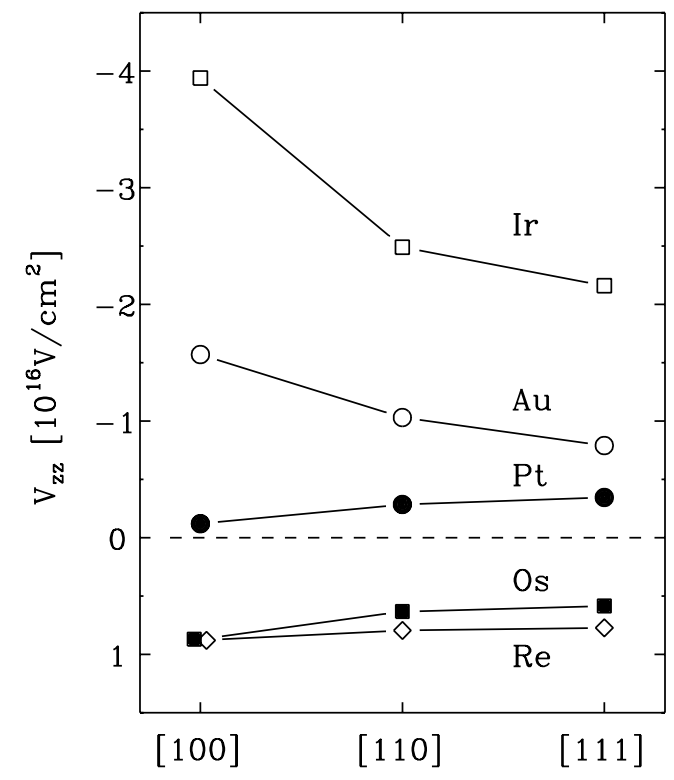

FIG. 3. EFG of Os, Re, Ir, Pt, and Au in Fe for $M \|$ [100], [110], and [111]. 


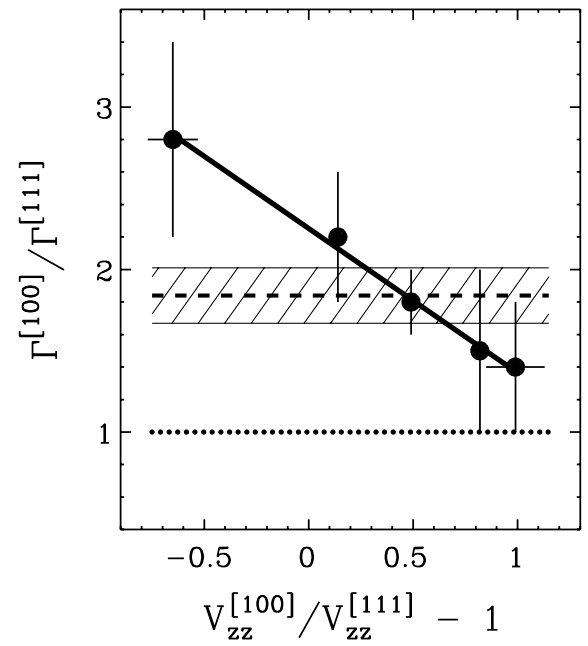

FIG. 4. Ratio of the EFG width $\Gamma^{([100])} / \Gamma^{([111])}$ vs EFG anisotropy $V_{z z}^{([100])} / V_{z z}^{([111])}-1$.

sign change. In the framework of the theoretical models of Refs. $[5,15]$ the spin-orbit EFG depends on the relative amplitudes of the five wave functions of the $5 d$ electrons at the impurity site $\left(\Gamma_{3}: x^{2}-y^{2}, 3 z^{2}-r^{2} ; \Gamma_{5}: x y, y z, z x\right)$ : For degenerate $\Gamma_{3}$ and $\Gamma_{5}$ states, the EFG is isotropic; assuming that only the $\Gamma_{5}$ states are relevant, an anisotropic EFG is expected, with $\left(V_{z z}^{[100]}-V_{z z}^{[111]}\right) / V_{z z}^{[111]}<0[5,15]$, i.e., opposite to the majority of the observed signs. In our opinion one of the most crucial deficiencies in the mentioned theoretical models could be the fact that the band structure of $5 d$ electrons at the impurity site has been neglected completely.

The different widths of the EFG distribution for $M \|$ [100], [110], and [111] could also be connected with details of the band structure. It cannot be due to [100]-lattice defects - these would produce a broadening for $M \|$ [100] which is not visible for $M \|$ [111] — since impurity nuclei with a defect in the near neighborhood do not contribute to the MAPON signal because of the different resonance frequency with respect to nuclei with an undisturbed surrounding. In principle, the EFG width could be connected with the following facts: The EFG's of pure $\Gamma_{3}$ and $\Gamma_{5}$ states are of the order of $10^{18} \mathrm{~V} / \mathrm{cm}^{2}$, i.e., 2 orders of magnitude larger than the observed EFG's. The "small" actual EFG's are due to the fact that large components with positive and negative signs nearly cancel. For complete quenching of the orbital momentum the amplitudes for the different components are so that the resulting EFG is zero. The spin-orbit interaction lifts the degeneracy of the involved states, entailing a nonzero EFG. Thus, however, small changes of the wave functions of the involved states may imply a relatively large variation of the EFG. Because of the different elastic constants of the Fe lattice for the [100] and [111] directions, (small) displacements of the atoms from their ideal lattice positions - these could be due to charge and/or spin density waves, possibly also due to a Jahn-Teller effect - are energetically favored along the [100], [010], and [001] directions. This would imply a broadening of the EFG width for $M \|$ [100], which, because of the magic angle of $55^{\circ}$, is invisible for $M \|$ [111].

In summary, we have observed that the anisotropy of the spin-orbit EFG of $5 d$ elements in Fe depends specifically on the properties of the probe atoms. Within the $5 d$ series the EFG anisotropy changes sign for Pt. In addition, the width of the EFG distribution depends on the direction of magnetization with respect to the crystallographic axes. The anisotropy of the EFG width seems to be correlated with the EFG anisotropy. These findings cannot be understood within the presently adopted theoretical models. Our results should stimulate new theoretical work for the understanding of EFG's in cubic ferromagnets, with a proper inclusion of the band structure at the impurity site.

We appreciate very much the effort which was put by the ISOLDE and Orsay groups into the development of the liquid $\mathrm{Pb}$ target. We also thank E. Smolic for experimental help. This work has been funded by the Deutsche Forschungsgemeinschaft (DFG) under Contract No. Ha 1282/3-3.

[1] M. Aiga and J. Itoh, J. Phys. Soc. Jpn. 31, 1844 (1971).

[2] R. L. Mössbauer, M. Lengsfeld, W.v. Lieres, W. Potzel, P. Teschner, F. E. Wagner, and G. Kaindl, Z. Naturforsch. 26A, 343 (1971).

[3] D. Salomon and D. A. Shirley, Phys. Rev. B 9, 29 (1974).

[4] P. D. Johnston and N. J. Stone, J. Phys. C 5, L303 (1972).

[5] M. Aiga and J. Itoh, J. Phys. Soc. Jpn. 37, 967 (1974).

[6] P. T. Callaghan, P. D. Johnston, W. M. Lattimer, and N. J. Stone, Phys. Rev. B 12, 3526 (1975).

[7] E. Hagn, K. Leuthold, E. Zech, and H. Ernst, Z. Phys. A 295, 385 (1980).

[8] P. C. Riedi and E. Hagn, Phys. Rev. B 30, 5680 (1984).

[9] M. Kawakami, H. Enokiya, and T. Okamoto, J. Phys. F 15, 1613 (1985).

[10] T. Dumelow, E. Hagn, P. C. Riedi, and E. Zech, Hyperfine Interact. 51, 915 (1989).

[11] G. Seewald, E. Hagn, E. Zech, D. Forkel-Wirth, A. Burchard, and ISOLDE Collaboration, Phys. Rev. Lett. 78, 1795 (1997).

[12] G. Seewald, thesis, TU Munich (unpublished).

[13] C. Demangeat, J. Phys. F 4, L64 (1974); J. Phys. F 5, 169 (1975).

[14] C. Demangeat, J. Phys. F 5, 1637 (1975).

[15] G. A. Gehring and H. C. W. L. Williams, J. Phys. F 4, 291 (1974).

[16] H. Ebert (private communication).

[17] P. T. Callaghan, P. J. Back, D. H. Chaplin, H. R. Foster, and G. V.H. Wilson, in Proceedings of the International Symposium on Nuclear Orientation and Nuclei far from Stability [Hyperfine Interact. 22, 39 (1985)].

[18] P. T. Callaghan, P. J. Back, and D.H. Chaplin, Phys. Rev. B 37, 4900 (1988).

[19] Th. Hilberath, St. Becker, G. Bollen, H.-J. Kluge, U. Krönert, G. Passler, J. Rikovska, R. Wyss, and ISOLDE Collaboration, Z. Phys A 342, 1 (1992). 